\title{
CHARACTERIZATIONS OF ALMOST PERIODIC TRANSFORMATION GROUPS ${ }^{1}$
}

\author{
W. H. GOTTSCHALK
}

Let $X$ be a uniform space, let $T$ be a multiplicative topological group, and let $T$ act as a transformation group on $X$.

A subset $A$ of $T$ is said to be (left) syndetic provided that $T=A K$ for some compact subset $K$ of $T$. The transformation group $T$ is said to be almost periodic on $X$ provided that if $\alpha$ is an index of $X$, then there exists a syndetic subset $A$ of $T$ such that $x A \subset x \alpha$ for all $x \in X$. If $x \in X$, then the transformation group $T$ is said to be locally almost periodic at $x$ provided that if $U$ is a neighborhood of $x$, then there exist a neighborhood $V$ of $x$ and a syndetic subset $A$ of $T$ such that $V A \subset U$. The transformation group $T$ is said to be locally almost periodic on $X$ in case $T$ is locally almost periodic at every point of $X$. If $x \in X$, then the transformation group $T$ is said to be locally weakly almost periodic at $x$ provided that if $U$ is a neighborhood of $x$, then there exist a neighborhood $V$ of $x$, a syndetic subset $A$ of $T$, and a compact subset $C$ of $T$ such that $y \in V$ implies the existence of a subset $B$ of $T$ for which $A \subset B C$ and $y B \subset U$. It is readily proved that if $x \in X$, then $T$ is locally weakly almost periodic at $x$ if and only if for each neighborhood $U$ of $x$ there exist a neighborhood $V$ of $x$ and a compact subset $K$ of $T$ such that $V T \subset U K$.

If $x \in X$, then the transformation group $T$ is said to be equicontinuous at $x$ provided that if $\alpha$ is an index of $X$, then there exists an index $\beta$ of $X$ such that $x \beta t \subset x t \alpha$ for all $t \in T$. The transformation group $T$ is said to be equicontinuous on $X$ in case $T$ is equicontinuous at every point of $X$. The transformation group $T$ is said to be $u n i$ formly equicontinuous on $X$ provided that if $\alpha$ is an index of $X$, then there exists an index $\beta$ of $X$ such that $x \beta t \subset x t \alpha$ for all $x \in X$ and all $t \in T$. It is readily verified that if $X$ is compact, then $T$ is uniformly equicontinuous if and only if $T$ is equicontinuous.

The transformation group $T$ is said to be distal on $X$ provided that if $x, y \in X$ with $x \neq y$, then there exists an index $\alpha$ of $X$ such that $(x t, y t) \notin \alpha$ for all $t \in T$.

We also consider $T$ to be a transformation group acting on $X \times X$ in the following manner: if $x, y \in X$ and if $t \in T$, then $(x, y) t$ is defined to be $(x t, y t)$.

Received by the editors July 12, 1955.

1 This research was supported by the United States Air Force through the Office of Scientific Research of the Air Research and Development Command. 

[2].

As a general reference for the notions occurring in this paper, see

LemMA 1. Let $X$ be compact and suppose there exists $x \in X$ such that $T$ is locally weakly almost periodic at $(x, x)$ but $T$ is not equicontinuous at $x$. Then $T$ is not distal on $X$.

Proof. Let $\Re$ be the neighborhood filter of $x$. Since $T$ is not equicontinuous at $x$, there exists an open neighborhood $U$ of the diagonal of $X \times X$ such that $(N \times N) T \nsubseteq U$ for every $N \in \Re$. Define $\mathcal{F}=\left\{(N \times N) T \cap U^{\prime} \mid N \in \mathfrak{\Re}\right\}$ where $U^{\prime}$ is the complement of $U$ in $X \times X$. In order to show that $T$ is not distal on $X$ it is enough to show that $\cap \mathcal{F} \neq \varnothing$. Since $\bar{F}=\{\bar{F} \mid F \in \mathcal{F}\}$ is a closed filter-base on the compact set $U^{\prime}$, it follows that $\cap \bar{F} \neq \varnothing$. We complete the proof by showing that each member of $\mathcal{F}$ contains some member of $\bar{F}$ whence $\cap \mathcal{F} \supset \cap \bar{F}$ and thus $\cap F \neq \varnothing$. Let $N \in \Re$. Choose a closed neighborhood $N_{1}$ of $x$ such that $N_{1} \subset N$. Since $T$ is locally weakly almost periodic at $(x, x)$, there exist a neighborhood $M$ of $x$ and a compact subset $K$ of $T$ such that $(M \times M) T \subset\left(N_{1} \times N_{1}\right) K$ whence cls $(M \times M) T$ $\subset\left(N_{1} \times N_{1}\right) K \subset(N \times N) T$ and $(N \times N) T \wedge U^{\prime} \supset \operatorname{cls}\left((M \times M) T \wedge U^{\prime}\right)$. This shows that every member of $\mathcal{F}$ contains some number of $\bar{F}$. The proof is completed.

ThEOREM 1. Let $X$ be compact. Then the following statements are pairwise equivalent:

(1) $T$ is almost periodic.

(2) $T$ is locally almost periodic on $X$ and $T$ is distal on $X$.

(3) $T$ is locally weakly almost periodic at every point of the diagonal of $X \times X$ and $T$ is distal on $X$.

Proof. It is known $[2,4.38]$ that if $X$ is compact, then $T$ is almost periodic if and only if $T$ is uniformly equicontinuous. It follows easily from this theorem that (1) implies (2). An independent proof that (1) implies (2) may also be given.

Suppose now that $T$ is locally almost periodic on $X$ and let $x \in X$. We show $T$ is locally weakly almost periodic at $(x, x)$. Let $U$ be a neighborhood of $x$. There exist a neighborhood $V$ of $x$ and a syndetic subset $A$ of $T$ such that $V A \subset U$. Let $K$ be a compact subset of $T$ for which $T=A K$. We conclude that $(V \times V) T \subset(U \times U) K$.

It is now clear that (2) implies (3). That (3) implies (1) is immediate from Lemma 1 . The proof of the theorem is completed.

We say that $X$ is a minimal orbit-closure under $T$ or simply that $X$ is minimal under $T$ in case $\overline{x T}=X$ for all $x \in X$. A discrete flow is a transformation group whose phase group $T$ is the additive group of 
integers with the discrete topology. A discrete flow is completely determined by a homeomorphism of the phase space $X$ onto itself.

None of the conditions in (2) or (3) of Theorem 1 is redundant. It is known $[2,12.63]$ that there exist compact metrizable zerodimensional locally almost periodic minimal orbit-closures under discrete flows which are not almost periodic. A simple example of a ring of concentric circles rotating at different rates about their common center shows that distal alone does not imply almost periodicity.

Let $x, y \in X$. The pair $(x, y)$ is said to be proximal under $T$ provided that if $\alpha$ is an index of $X$, then there exists $t \in T$ such that $(x t, y t) \in \alpha$. Of course, there exists a pair of distinct points of $X$ which is proximal under $T$ if and only if $T$ is not distal on $X$. The pair $(x, y)$ is said to be syndetically proximal under $T$ provided that if $\alpha$ is an index of $X$, then there exists a syndetic subset $A$ of $T$ such that $(x a, y a) \in \alpha$ for all $a \in A$.

Lemma 2. Let $X$ be compact, let $x, y \in X$, let $(x, y)$ be proximal under $T$, let $\alpha$ be an index of $X$, and let $K$ be a compact subset of $T$. Then there exists $t \in T$ such that $(x t k, y t k) \in \alpha$ for all $k \in K$.

Proof. Since $X \times K$ is compact, the phase projection $\pi: X \times T \rightarrow X$ defined by $\pi(x, t)=x t$ is uniformly continuous on $X \times K$. Hence there exists an index $\beta$ of $X$ such that $\left(x_{1}, x_{2}\right) \in \beta$ implies $\left(x_{1} k, x_{2} k\right) \in \alpha$ for all $k \in K$. Choose $t \in T$ such that $(x t, y t) \in \beta$. The conclusion follows.

Leмma 3. Let $X$ be compact, let $x, y \in X$, and let $T$ be locally almost periodic at $x$. Then $(x, y)$ is syndetically proximal if and only if $(x, y)$ is proximal.

Proof. The necessity is obvious. We prove the sufficiency. Suppose $(x, y)$ is proximal. Let $\alpha$ be an index of $X$. Choose a neighborhood $U$ of $x$ such that $U \times U \subset \alpha$. There exist a neighborhood $V$ of $x$ and a syndetic subset $A$ of $T$ such that $V A \subset U$. Choose an index $\beta$ of $X$ such that $x \beta^{2} \subset V$. There exists a syndetic subset $B$ of $T$ such that $x B \subset x \beta$. Let $H$ be a compact subset of $T$ such that $T=B H$. By Lemma 2 there exists $t_{0} \in T$ such that $\left(x t_{0} h^{-1}, y t_{0} h^{-1}\right) \in \beta$ for all $h \in H$. Now $t_{0}=b_{0} h_{0}$ for some $b_{0} \in B$ and some $h_{0} \in H$. Since $t_{0} h_{0}^{-1}=b_{0}$, it follows that $\left(x b_{0}, y b_{0}\right) \in \beta$. Altogether we now have $x b_{0} \in x \beta \subset V$ and $y b_{0} \in x b_{0} \beta \subset x \beta^{2} \subset V$ whence $x b_{0}, y b_{0} \in V$ and $\left(x b_{0} a, y b_{0} a\right) \in U \times U \subset \alpha$ for all $a \in A$. Thus $t \in b_{0} A$ implies $(x t, y t) \in \alpha$. Since $b_{0} A$ is a syndetic subset of $T$, the proof is completed.

Theorem 2. Let $X$ be compact and let $T$ be locally almost periodic on $X$. Then there exists a pair of distinct points of $X$ which is syndetically proximal under $T$ if and only if $T$ is not equicontinuous on $X$. 
Proof. Use Theorem 1 and Lemma 3.

Let $x, y \in X$. The pair $(x, y)$ is said to be (totally) asymptotic under $T$ provided that if $\alpha$ is an index of $X$, then there exists a compact subset $K$ of $T$ such that $(x t, y t) \in \alpha$ for all $t \in T-K$.

A study (see below) of the example in [1] will reveal that the phrase "syndetically proximal" in Theorem 2 cannot be replaced by "asymptotic," even though it is assumed in addition that $X$ is a compact plane set, $T$ is a discrete flow, $X$ is minimal under $T$, and $T$ is regularly almost periodic at some points of $X$.

We indicate briefly why this example cannot possess even a unilaterally asymptotic pair of distinct points. We adopt here the notation used in [1] and we assume familiarity with the paper. First of all, $T$ is locally almost periodic since $X$ is minimal under $T$ and some points of $X$ are regularly almost periodic. This is an application of a general theorem (cf. $[2 ; 5.24]$ ). Since $f$ has equicontinuous powers, no two components of $X$ are even proximal. Consequently, any asymptotic pair of points belonging to $X$ would have to both lie in the same component. Now the nondegenerate components cannot have lengths which tend to zero under iteration of $T$. This is shown as follows:

Let $x$ be the point $\left(3^{0}, 3^{0}+3^{1}, 3^{0}+3^{1}+3^{2}, \cdots\right)$ of $A$. The component $V$ of $X$ which lies over $x$ is the longest and indeed has length 1. Let $\alpha_{0}, \cdots, \alpha_{n}$ be an arbitrary finite sequence made up of $0,1,2$. If the map $f$ is applied $\left(\alpha_{0}-1\right) 3^{0}+\left(\alpha_{1}-1\right) 3^{1}+\cdots+\left(\alpha_{n}-1\right) 3^{n}$ times to $x$, then the point $\left(\alpha_{0} 3^{0}, \alpha_{0} 3^{0}+\alpha_{1} 3^{1}, \ldots\right)$ is obtained. Consequently the images of $V$ constitute all nondegenerate components of $X$ and are of length $1 / 2$ infinitely often in both directions.

\section{BiBLIOGRAPHY}

1. E. E. Floyd, A nonhomogeneous minimal set, Bull. Amer. Math. Soc. vol. 55 (1949) pp. 957-960.

2. W. H. Gottschalk and G. A. Hedlund, Topological dynamics, Amer. Math. Soc. Colloquium Publications, vol. 36, 1955.

University of Pennsylvania 